\title{
Modificación del injerto libre En agenesia vaginal
}

FERNANDO DEL CORRAL G., M.D.(*) AUGUSTO BOTERO T.

La Agenesia Vaginal fue descrita por primera vez en 1.572 por Realdus Columbus(1). Quizá uno de los manuscritos que revisa más completamente el problema de Agenesia Vaginal y su corrección, corresponde a Capraro y Gallego (1), quienes revisan el aspecto quirúrgico y emocional del paciente y presentan su experiencia personal en cincuenta casos.

Mi primer contacto en este problema lo tuve en el año de 1.959, cuando trabajando con Edwin Hamblen (2), uno de los pioneros de la Ginecología Endocrina, aprendí la implicación de las amgüedades genitales en el aspecto emocional del paciente, al mismo tiempo que su tratamiento hormonal y quirúrgico.

En los casos de Hermafroditismo y pseudo - hermafroditismo, al paciente lo descubre tempranamente el médico o los familiares, y su tratamiento comienza generalmente desde el nacimiento.

En los casos de falla embriológica de los canales de Müller, a veces la primer llamada de alerta es la falla en aparición de la menstruación en la paciente adolescente, y sólo hasta este momento la paciente crea conciencia de su problema.

En uno y otro caso; el aspecto emocional debe ser considerado y manejado oportunamente sin excluir todo el estudio endocrino correspondiente.

Los pacientes para Vaginoplastia pueden ser considerados dentro de los siguientes grupos:

1. Paciente con cariotipo o fenotipo femeninos, con útero funcional y agenesia vaginal.

2. Paciente con las mismas características

(*) Presidente Sociedad Colombiana de Médicos Endoscopistas.

Presidente Sociedad Vallecaucana de Obstetricia y Ginecología. femeninas anteriores, con ausencia completa del útero y vagina.

3. Paciente similar al número dos, pero con alteraciones del tracto urinario: agenesia unilateral de riñón, riñón único en herradura, riñón pélvico unilateral, reduplicación de canales escretores, etc y/o malformaciones del sistema esquelético (Síndrome de Rokytansky - Küster . Hauser).

4. Hermafroditismo verdadero o pseudohermafroditismo masculio o femenino.

5. Transexualismo Masculino.

6. Desaparición traumática de vagina, extirpación quirúrgica por cáncer, u obliteración por irradiación.

Cualquiera que sea el caso, el paciente debe ser estudiado cuidadosamente, incluyendo el examen físico completo, estudio endocrinológico, psiquiátrico y citogenético; además de estudio radiográfico que incluye pielograma y también una comprobación por Laparoscopia.

TECNICAS QUIRURGICAS.

\section{PROCEDIMIENTO NO OPERATORIO DE} FRANK.

Este consiste en la formación de vagina por presión mediante el uso de bujías que ejercen presión constante entre el espacio recto -vesical (1), (3).

Este procedimiento requiere paciencia extrema por parte del paciente, y sus resultados solo pueden observarse a muy laryo plazo.

\section{I'ECNICA DE WHARTON}

Consiste en la tunelización quirúrgica del espacio recto-vesical, sin colocación de 
injerto, utilizando únicamente el molde.

Este procedimiento al igual que el anterior, tiene el inconveniente de ser muy demorado, esperando una lenta epitelización de la mucosa del introito hacia adentro, con la consiguiente infección secundaria inevitable.

\section{PROCEDIMIENTO DE MC INDOE(9).}

Exije la tunelización como en la Técnica de Wharton, y la aplicación de un molde cubierto de piel (Dermo -epidérmico), tomado de la región del muslo ó glútea. Los defectos dejados por el injerto son obvios y demorados en su cicatrización, la que se realiza por segunda intención.

Es muy frecuente la infección secunda- ria en el tejido de granulación. Las dilataciones posteriores son imperativas y deben ser frecuentes para evitar el encogimiento del injerto y la obliteración del espacio(1).

\section{TEJIDOS INTR AABDOMINALES.}

Este procedimiento es similar al anterior. Se utiliza un segmento del intestino delgado como la Técnica de Cognat (4), o con intestino grueso como en la descrita por Pratt(5).

Esta técnica lleva implícitos los riesgos de infección del injerto, de por sí contaminado. La secreción mucoide propia de uno y otro tejido es por sí solo un inconveniente, además, es un procedimiento de riesgo quirúrgico no menospreciable, cual es la
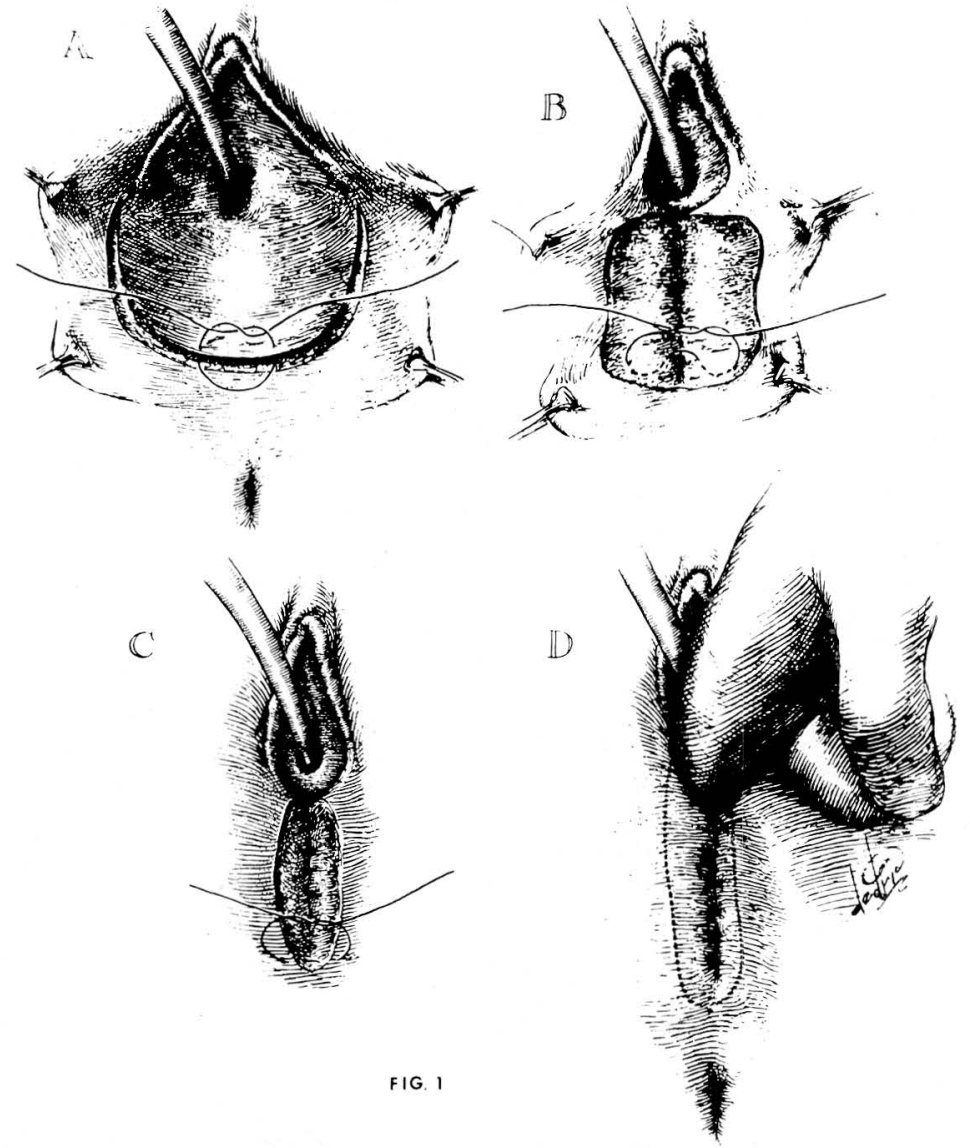
laparotomía con reseción intestinal y anastomosis.

También puede ser utilizado el peritoneo como lo propone Rothman(6).

\section{TRASPLANTE DE VAGINA.}

Utilización de vagina obtenida de un donante (generalmente la madre o una hermana), como lo propuesto por Papanicolau (7).

Obvios son los inconvenientes con la posibilidad de rechazo inmunológico y las molestias ocasionadas a la donante, con la consiguiente pérdida del epitelio vaginal.

\section{TECNICA DE WILLIAMS}

Es una vulvo-vaginoplastia que utiliza los tejidos de los labios menores(8). Esta técnica fabrica algo similar a una bolsa de canguro.

Ha sido modificada por el Grupo de Buffalo (fig. 1 y 2); el eje de la vagina aunque distorsionado puede ser corregido por el uso periódico de dilatadores y con el coito (1).

Sus ventajas son el acortamiento del tiempo quirúrgico con menor pérdida de sangre y las no secuelas de cicatrización de las otras técnicas de injerto libre.

\section{NUESTRA TECNICA}

Basicamente es la Técnica de Mc Indoe, pero en lugar de tomar injertos libres de piel del muslo o la región glútea, los que para ser efectivos deben ser dermo-epidérmicos completos, dejando defectos visibles por su cicatrización tardía.

Usamos piel obtenida de abdómen en la región intermedia entre pubis y ombiigo, evitándo la región pilosa del Monte de Venus.

El colgajo se obtiene en forma de rombo de $28 \mathrm{cms}$ de longitud por 6 o $7 \mathrm{cms}$ de ancho en su parte media (Figura 3 ). Una vez obtenido el injerto, el cierre de piel se

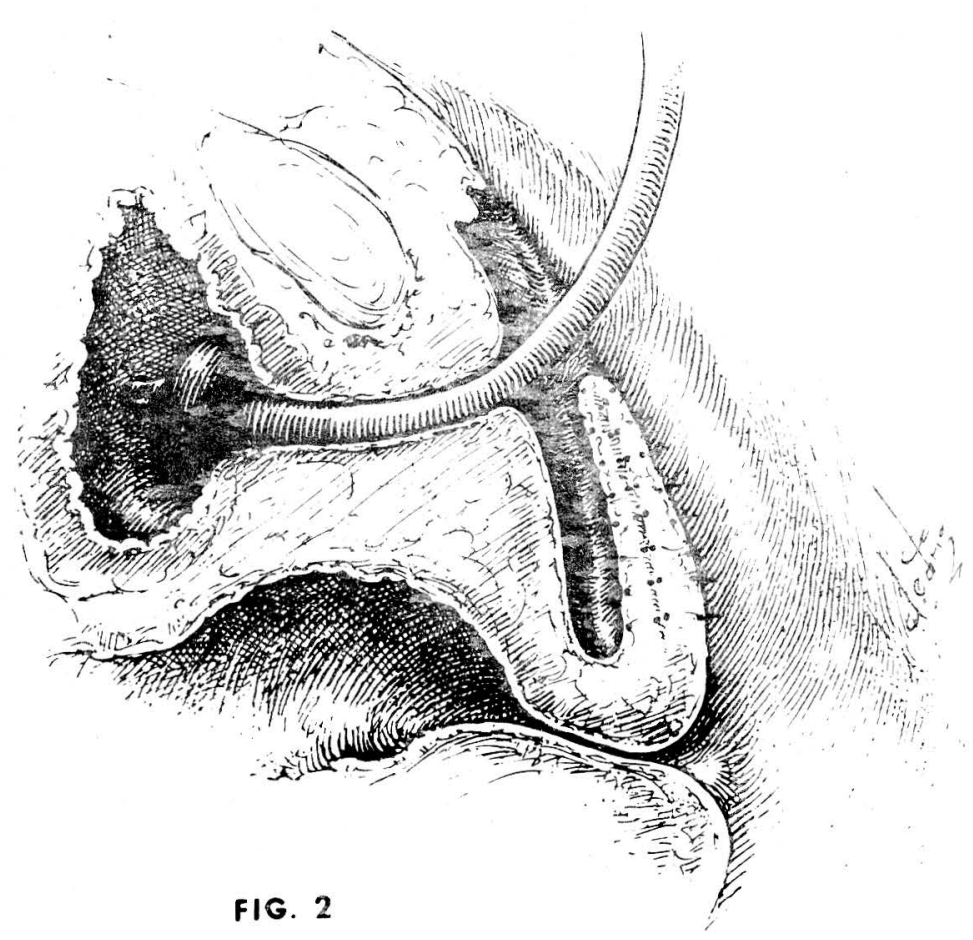



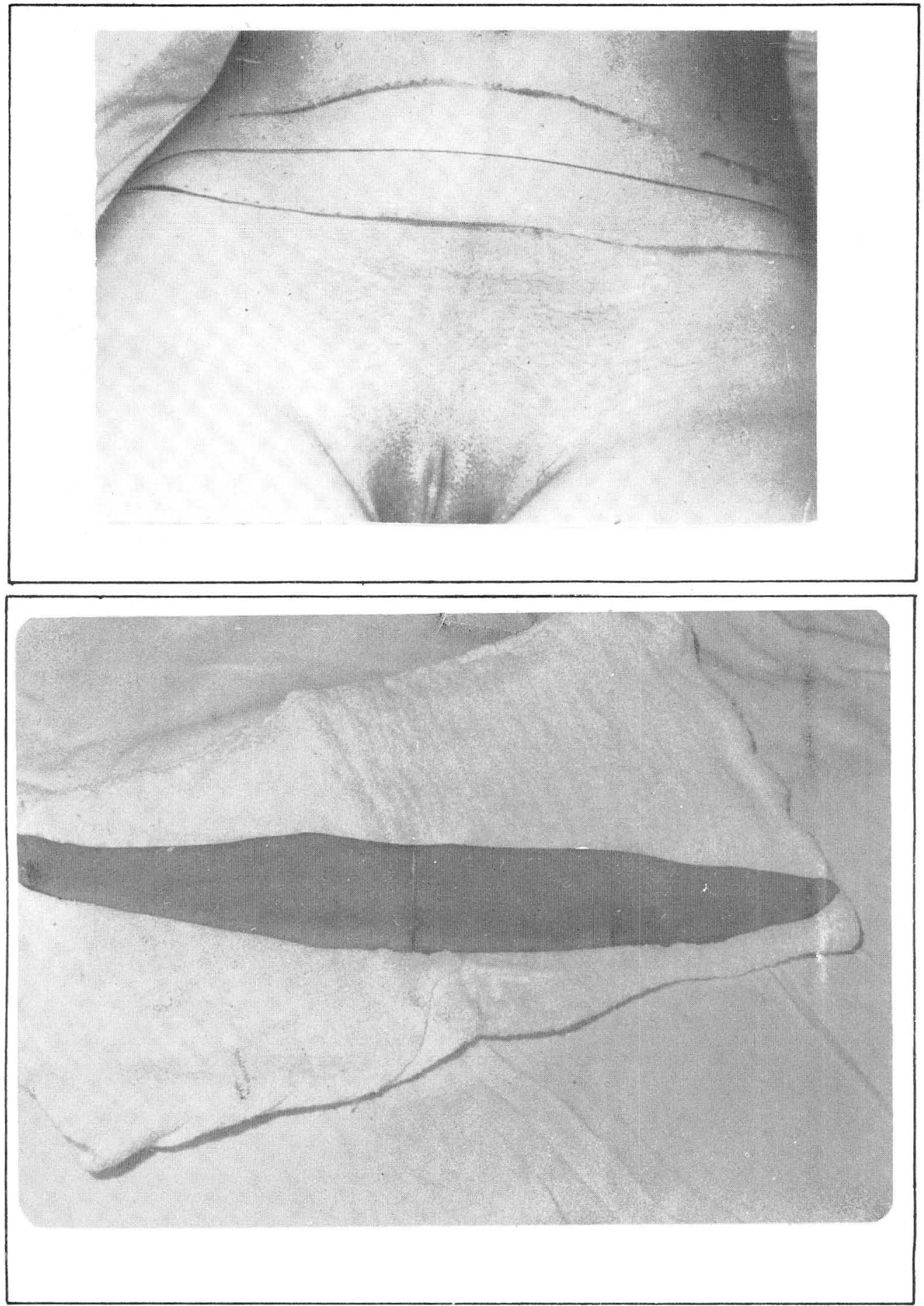


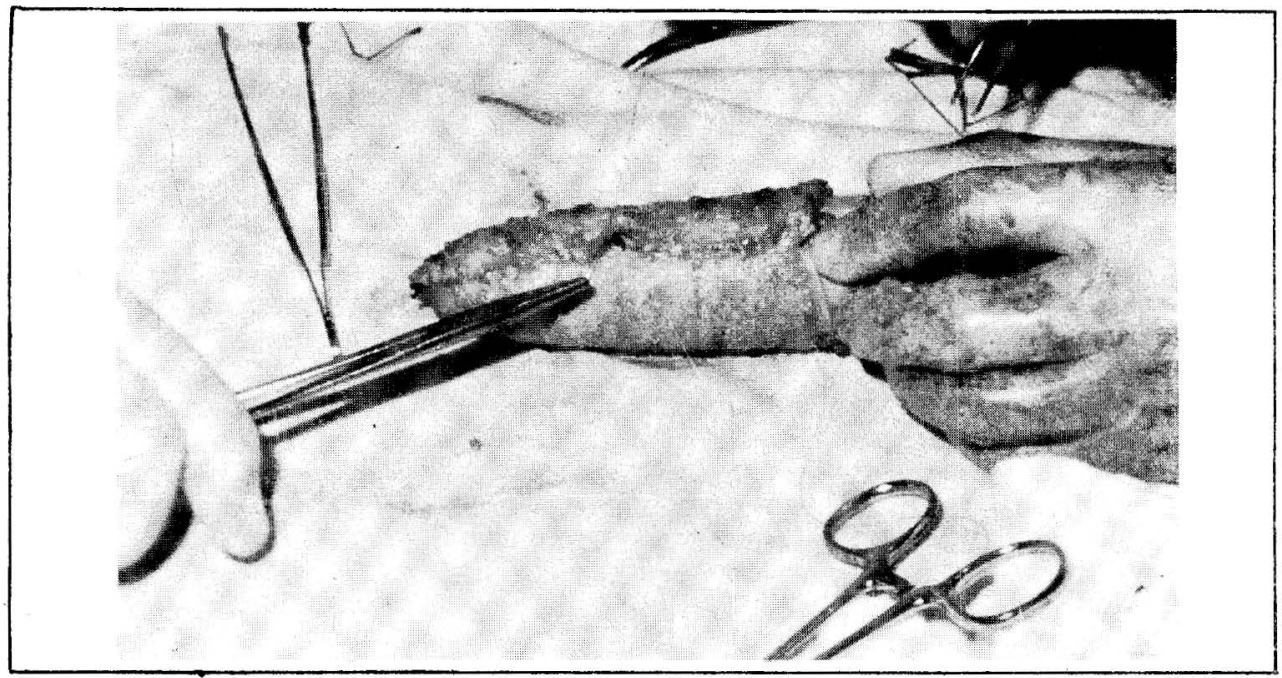

hace por deslizamiento.

Como no se sacrifica la grasa subcu tánea a la toma del injerto, la cicatrización se hace por primera intención, rápidamente, de-

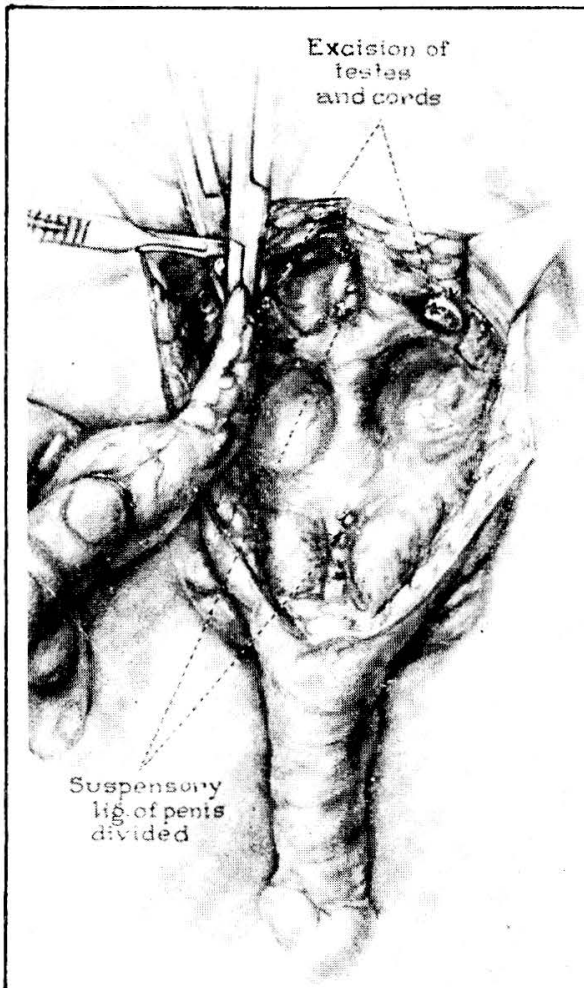

bido a su excelente vascularización, sin dejar ninguna depresión o solución de continuidad.

Una vez obtenida la piel, se secciona en

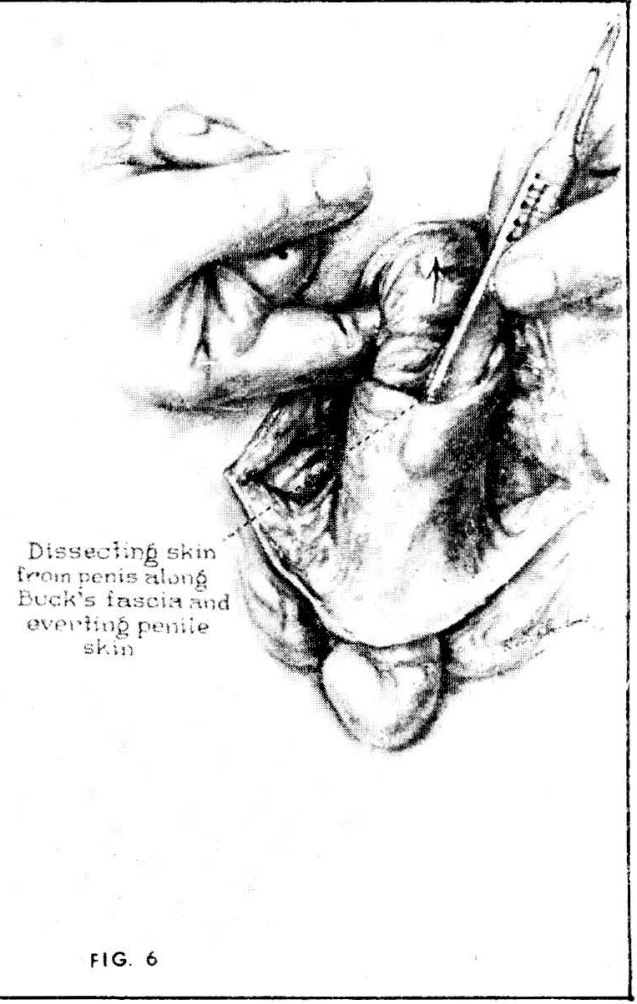




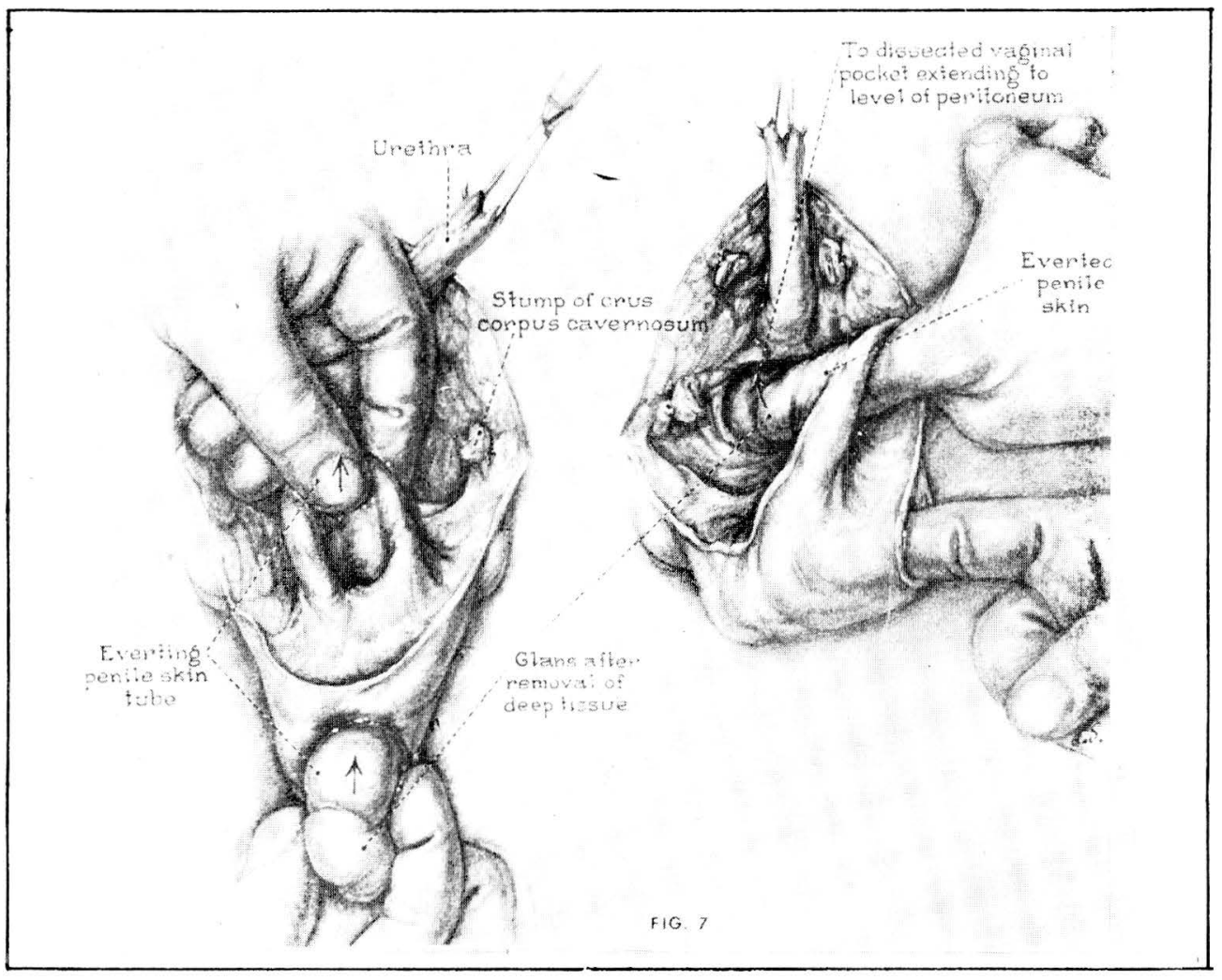

su parte media formando dos triángulos (Figura 4), los cuales se suturan sobre el molde con material no absorbible (Dermalon 00), (Figura 5). Esto permite que el normal encogimento de la piel de todo injerto, sea menor, por las aberturas laterales de la piel.

Creemos que esta técnica de toma de injerto es muy bien aceptada por el paciente, ofreciéndo solo una cicatriz transversal similar a las obtenidas en una lipectomía medio baja.

\section{TRANSEXUALISMO}

En esta entidad también llamada hermafroditismo siquico, solamente tenemos eperiencia en el anejo del tipo masculino.

Antiguamente se manejaba únicamente con la amputación del phallus; posteriormente se corrigieron por el procedimiento de $\mathrm{Mc}$ Indoe(9), con injertos de piel libre (como se mencionó anteriormente), cubiertos sobre molde de madera, plástico o de silastic, dándola al transexual, la oportunidad de una vagina funcional.

\section{TECNICA DE FORTUNOFF (10)}

Ha sido modificada por el Grupo del John's Hopkins, y descrita por Jones (11), utilizando tejido escrotal, para la formación de la vagina.

\section{TECNICA DE EDGERTON(12)}

Este autor ilustra en forma muy explícita en su publicación, la técnica con la utilización de un injerto pediculado hecho con la piel del pene con conservación del glande. (Artículo del cual reproducimos la figura correspondiente, Figuras 6 y 7 ).

Nosotros, siguiento la misma técnica, utilizarros en igual forma la piel del pene, previo deslizamiento y desprendimiento 
de la piel de la región abdominal, modificando la toma del colgajo, mediante incisión semilunar de concavidad inferior, de tal manera que en lugar de quedar adherido al pedículo de la porción inferior del pene, queda adherido a la porción superior.(Figuras 8 y 9 de publicación del autor)(13)

\section{CASUISTICA}

En este escrito queremos esbozar solamente los resultados obtenidos en la creación de vagina artificial, con las modificaciones:

A. A la técnica de Mc Indoe con injerto libre de pared abdominal, en ocho casos con resultados satisfactorios, todos en pacientes jóvenes, con agenesia vaginal.

De las ocho pacientes; cuatro presentaban alteraciones concomitantes del tracto urinario con agenesia unilateral de riñón y una con riñón pélvico único.

En todos estos casos las pacientes presentaban un desarrollo somático femenino satisfactorio, debido a la presencia de ovarios funcionalmente normales.

Las edades de las pacientes fueron en promedio 24 años, y todas deseaban iener actividad sexual.

El molde de estas pacientes fue retirado

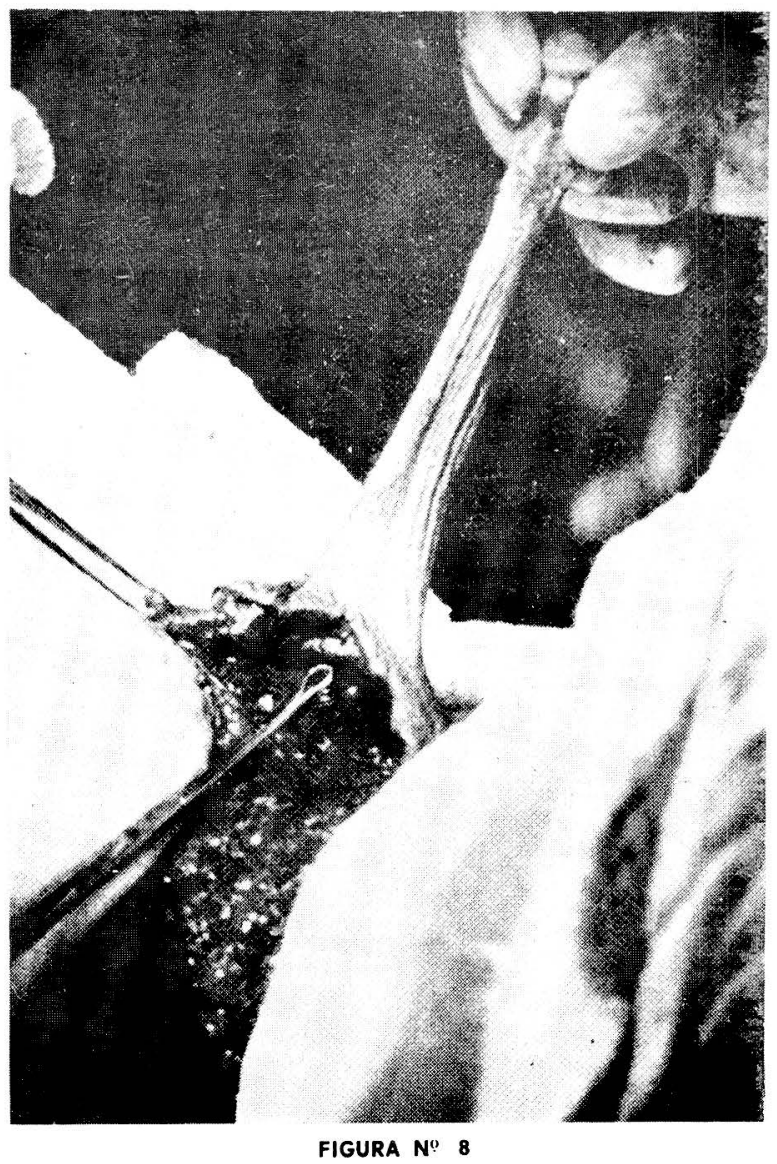




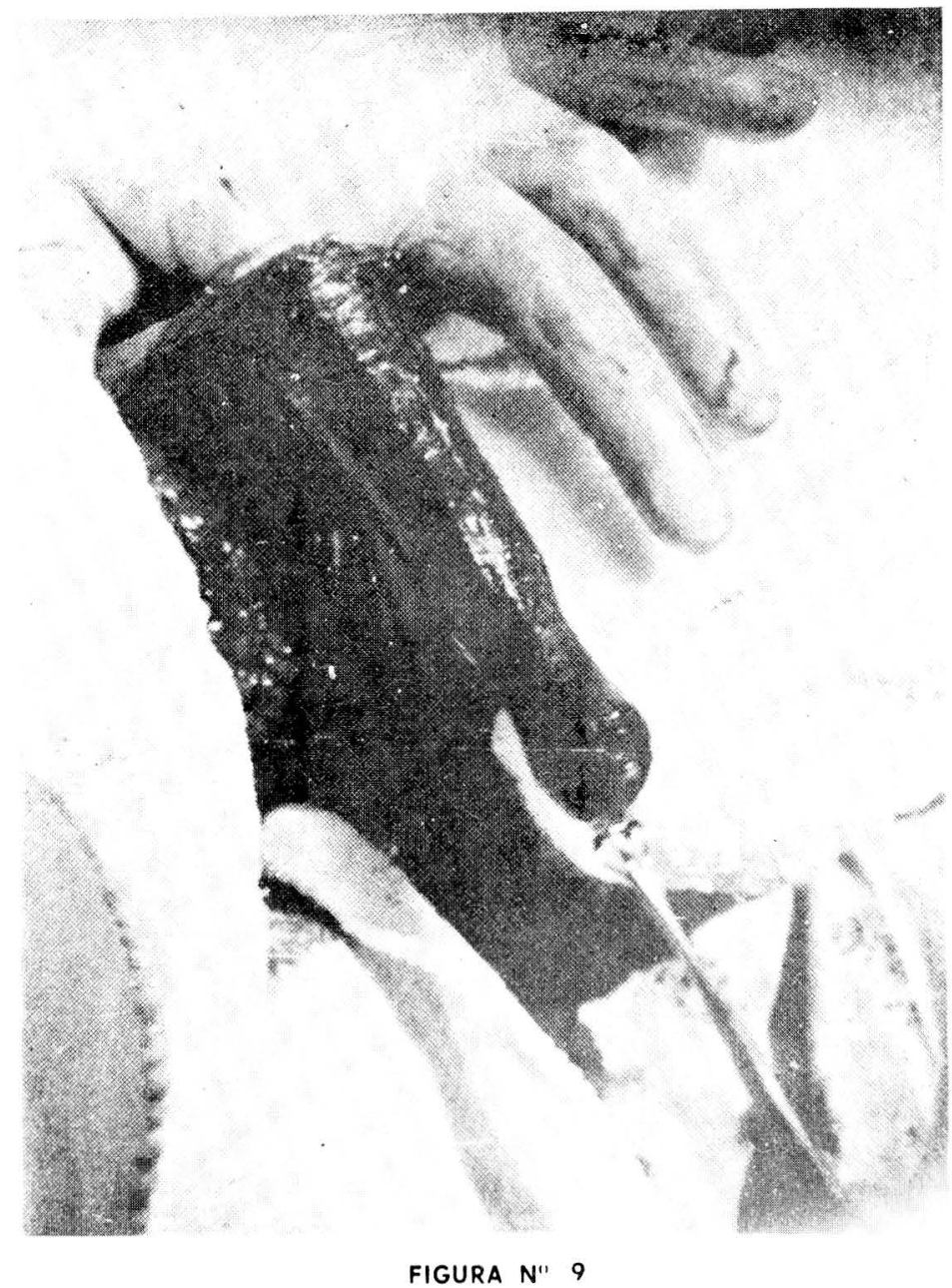

al octavo día del post-operatorio, tiempo durante el cual tuvieron un cateter vesical a permanencia, recibiendo además dieta líquida o blanda sin residuos, y una medicación antiperistáltica.

Los resultados fueron satisfactorios en todas las pacientes, las cuales continuaron utilizando periódicamente el molde por lo menos durante media hora al día hasta regularizar sus relaciones sexuales

B. Modificación a la Técnica de Edgerton en el manejo de vagina artificial, en dos pacientes con transexualismo masculino, uno de los cuales fue publicado con anterioridad.(13).(Figuras 8 y 9 ).

Los dos casos de transexualismo masculino operados por la Técnica de Edgerton modificada por nosotros, tienen actualmente una vagina funcional.

\section{COMENTARIO}

Es importante conocer los diferentes aspectos en el manejo del paciente que requiere una construcción de neo-vagina. 
La época más oportuna para realizar la intervención es una vez terminada la pubertad y el desarrollo corporal,

En esta forma, la edad cronológica del paciente permite también una mejor adaptación al procedimento, así como la colaboración necesaria para su adiestramiento en la utilización del molde $\mathrm{y} / \mathrm{o}$ el coito.

\section{RESUMEN}

- Se presenta una revisión escueta de las técnicas descritas para la creación de neovagina, haciendo un análisis somero de sus beneficios y ventajas.

Se presenta la modificación del injerto libre con resultados excelentes en ocho pacientes con Agenesia Vaginal y se ilustra la técnica correspondiente.

\section{SUMMARY}

A review is made of the techniques described for neo-vaginal creation through an analysis of its benefits and advantages.

A modification is introduced in free graft yielding excellent results in 8 pacients with vaginal agenesia; and the corresponding technique is illustrated.

\section{BIBLIOGRAFIA}

(1) Cited by Caparo V.J., Gallego M.B. Current Developments., Am. J. Obst and Gynec. 124 No. 1: 98 - 107., January 1976.

(2) Hamblen Edwing - Personal Comunication - Duke University - Medical Center, Durham, North Carolina., 1959.
(3) Wabrek, A.J., Millard R.P., Wilson W.B. and Pion R.J.: Creation of a Neovagina by Frank monoperative Method., Obst - Gynec 37: 408., 1971.

(4) Cognat M. Vuillard P. and Soustelle J: Traitment chirurgical de l'abscense congenitale du vagin., Int. Surg., 52: 494., 1969.

(5) Pratt J.H.: Vaginal ateresia corrected by use of small and large bowel, Clin. Obst. and Gynec., 15: 639., 1972.

(6) Rothman D.: The use of peritoneum in the construction of a vagin., Obstet Gynecol, 40: 835., 1972 .

(7) Papanicolau N.: Succesful vaginal transplant, mother to daughter., Obstet. Gynecol News., 8: 1., 1973.

(8) Williamns E.A.: Congenital absence of the vagina. A simple operation for its relief., J. Obst. Gynecol Br. Commown. 75:511., 1964.

(9) Me Indoe A.: Techniques for vaginoplasty., J. Obst Gynec Brit., Emp. 51 :24., 1944.

(10) Fortunoff S. et Al.: Vaginoplasty technique for female pseudohermaphrodites., Surg Gynec. and Obst., 118:545., 1964.

(11) Jones H., Shirmer H. and Hoopes J.: A sex convertion operation for males with transexualismo. Am. J. Obst and Gynecol., 100:101, 1968 .

(12) Edgerton M.T., Bull J.: Surgical construction of the vagina and labia in males transexual. Plastic and reconstruct. Surgery Vol 46 No. 6.,: 529 - 539., Dec. 1970.

(13) Del Corral F.: Transexualismo Masculino Reasignación del sexo por cirugía. Revista Colombiana de Obstretricia y Ginecología., Volúmen XXIII., No. 2:101 - 109., Marzo - Abril., 1972. 